Research Article

\title{
Investigating the Working Efficiency of Typical Work in High-Altitude Alpine Metal Mining Areas Based on a SeqGAN-GABP Mixed Algorithm
}

\author{
Ning Hua, ${ }^{1}$ He Huang $\mathbb{D},{ }^{2}$ and Xinhong Zhang $\mathbb{D}^{1}$ \\ ${ }^{1}$ Xinjiang Institute of Security Science and Technology, Urumqi, Xinjiang 830000, China \\ ${ }^{2}$ College of Mechanical Engineering, Xinjiang University, Urumqi, Xinjiang 830047, China
}

Correspondence should be addressed to Xinhong Zhang; hh_xnxs@163.com

Received 26 March 2021; Revised 10 June 2021; Accepted 28 June 2021; Published 11 August 2021

Academic Editor: Valeria Vignali

Copyright (c) 2021 Ning Hua et al. This is an open access article distributed under the Creative Commons Attribution License, which permits unrestricted use, distribution, and reproduction in any medium, provided the original work is properly cited.

\begin{abstract}
Man-machine efficacy evaluations of typical work in the safe mining of high-altitude alpine metal mines are associated with fuzziness, multiple indexes, and large subjective components. This results in difficulties in the prediction of the typical work efficiency in high-altitude alpine metal mining areas. In this study, ergonomic theory was applied to establish the evaluation index system of typical work efficiency in high-altitude alpine metal mining areas by studying the cooperative relationship between operators, working machines, working environment, and design variables. First, we investigated the collaborative relationship between workers, operating machinery, operating environment, and design variables in order to establish the evaluation index system of typical work efficiency in high-altitude alpine metal mining areas. Second, principal component analysis (PCA) was integrated with the fusion entropy weight method to (i) analyze the coupling correlation and overlapping effects between the factors influencing efficiency at different altitudes and (ii) to determine the key influencing factors. Third, a model based on the sequence generative adversarial network genetic algorithm backpropagation (SeqGAN-GABP) hybrid algorithm was established to predict the trends in the operating efficiency of typical work types in high-altitude alpine metal mining areas. Finally, three high-altitude alpine metal mines in Xinjiang were selected as representative examples to verify the proposed framework by comparing it with other state-of the art models (multiple linear regression prediction model, backpropagation (BP) neural network model, and genetic algorithm back propagation (GA-BP) neural network model). Results determine the average relative error of each model as $2.74 \%, 1.97 \%, 1.29 \%$, and $1.02 \%$, respectively, indicating the greater accuracy of our proposed method in predicting the efficiency of typical work types in high-altitude alpine mining areas. This study can provide a scientific basis for the establishment of mining safety judgment standards in high-altitude alpine areas.
\end{abstract}

\section{Introduction}

China possesses a highly developed mineral resource industry and the energy and production materials typically originate from mineral resources [1]. However, the majority of highquality mineral resources are distributed in high-altitude areas in the west. Due to limitations of immature production technology, the development of mineral resources in highaltitude areas has been slow. In recent years, the innovation of mining technology has upgraded mining equipment and improved infrastructure in the high-altitude alpine areas of the west. Moreover, the mining of high-altitude alpine metal mines in the west is included in the government's schedule [2]. Highaltitude alpine areas are rich in metal mineral resources, yet they are also accompanied by extreme environmental characteristics such as low pressure and hypoxia, cold and dry weather, and poor geological conditions. This consequently leads to poor mining conditions, complex disaster mechanisms, limited safety criteria, and difficulties in the quantitative analysis of operating efficiency [3]. Therefore, the ability to accurately estimate the operating efficiency of high-altitude alpine mining areas and predict its laws plays a key role in the rationality of mining safety decisions and has become a key problem for mining in high-altitude alpine mining areas. 
The special working environment in high-altitude alpine areas has a direct impact on the work efficiency of personnel and equipment, resulting in complex production factors and continuous labor in the mines. Thus, the assessment of work efficiency proves to be a complicated task [4]. Restricted by the special geographical environment of high-altitude alpine mining areas, as well as the multiple and complex influencing factors of the operating efficiency, current research on the prediction of mining operating efficiency under extreme conditions is limited. However, numerous studies have been performed on other forecasting problems, providing reference for the methodology adopted in this study.

The theory of ergonomics can determine the relationship between people, equipment, and environment. It plays a key role in improving the efficiency of personnel operating equipment and can provide a new perspective for the evaluation of operating efficiency under complex working conditions [5]. Numerous studies have been performed on the efficiency of construction operations in high-altitude areas based on operators, equipment, environment, and in particularly, factors that affect work efficiency for practitioners. For example, Egger et al. [6] discussed the influence of highaltitude physical labor on the quality of cardiopulmonary resuscitation. Qiulin et al. [7] explored the management strategies of unsafe behavior from mine employees. Anamika et al. [8] studied the relationship between intermittent atmospheric hypoxia and adaptive changes in altitude. Qiujun et al. [9] discussed the hypoxia status and oxygen supply effect of high-altitude tunnel workers and classified the human body's hypoxia hazard level under high-altitude working conditions. Xin et al. [10] conducted a graded evaluation of the physical labor intensity of tunnel operators under highaltitude conditions. Reza et al. [11] compared acute and nonacute altitude sickness headaches. Moreover, studies have investigated the factors affecting the operating efficiency of equipment. For example, Yang et al. [12] focused on the power recovery and gas pressurization system of high-altitude diesel engines. Blancher et al. [13] studied the influence of altitude on syringe infusion pumps. Yucheng and Yumin [14] evaluated the working performance of mine axial fans under high altitudes. Research on factors affecting work efficiency in the working environment include the work of Davis et al. [15], who evaluated the impact of living altitude on cognitive and psychomotor functions. Moreover, Johnson et al. [16] conducted a large-scale mercury-human health risk assessment for miners and villagers in an ingenio mining area in Bolivia. However, these studies are all analyzed from a single factor of production. Studies on the analysis of high-altitude mining from the perspective of ergonomics are limited. Ning et al. [17] evaluated the man-machine efficiency of a plateau mine based on the fuzzy analytic hierarchy process, while Guo et al. [18] analyzed the system dynamics of man-machine effectiveness in plateau mines. The majority of studies on the mining operations of high-altitude alpine metal mines consider single factors (e.g., the operator, equipment, or environment) as the key influence of mining activities. At present, research on the influence of the overall coupling effect between human and machine environments on mining operations is lacking.
Artificial neural networks (ANNs) have demonstrated a strong nonlinear fitting ability for prediction problems with strong disturbances and complex mechanisms and are able to establish the relationship between the input and output for unknown function relationships [19, 20]. ANNs are commonly used for scientific prediction problems in other fields. For example, Niu [21] established an artificial neural network model to determine the performance of diesel engines, while Das et al. [22] employed a hybrid neural network model to predict stock prices. Othman et al. [23] estimated the Bitcoin market price based on an ANN using symmetrical fluctuation information. Similarly, Natarajan et al. [20] established an ANN for a particle swarm optimization algorithm and applied it to life predictions. Banadkooki et al. [24] established an ANN-based hybrid model and an ant-lion optimization algorithm to predict suspended sand load. Wu and Lei [25] employed vibration signal analysis and artificial neural network methods to predict the surface roughness of milling. Wang et al. [26] proposed a new ICPSO-BPNN method, which combines the improved chaotic particle swarm optimization (ICPSO) with the backpropagation neural network (BPNN) to predict the monthly travel demand.

Among the numerous existing neural network prediction methods, BP neural networks have strong nonlinear mapping and generalization capabilities, as well as a high degree of self-learning and adaptivity. Thus, BP neural network-based methods are commonly employed to predict various engineering problems. In a study on the problem of magnetic exploration, Dihong et al. [27] predicted the comprehensive performance of concrete via BP neural networks. Wenju et al. [28] established a BP-based model to predict the buried depth of magnetic bodies. However, single BP neural networks are associated with several prediction defects, such as the lack of effective methods in the parameter selection and easily falling into the local optimum. Genetic algorithms can achieve global optimization, which can overcome the susceptibility of BP neural networks to fall into local optimums, thus increasing the prediction accuracy. Moreover, artificial neural network prediction methods require an extensive amount of experimental data, which can be difficult to obtain.

Therefore, based on the aforementioned literature, in the current study, we employ ergonomics theory to establish an operation efficiency evaluation index system for typical work types in high-altitude alpine mining areas. Principal component analysis (PCA) and the entropy weight method are fused to analyze the coupling correlation and overlapping influence of numerous factors affecting efficiency at different elevations and to determine the key indexes affecting operating efficiency under high-altitude alpine conditions. The data volume is expanded based on the sequence generative adversarial network (SeqGAN). A neural network model (SeqGAN-GABP hybrid algorithm model) is established based on the optimization of the generative adversarial network (GAN) and the genetic algorithm (GA) for backpropagation (BP) to predict the trends in the typical work efficiency in high-altitude alpine metal mines. Taking three high-altitude alpine metal mines in Xinjiang as typical 
examples of work involving air drillers, an evaluation index system for air drill operations is established based on the proposed hybrid algorithm. The key factors influencing efficiency are then obtained and the variations in the pneumatic drill operations are subsequently analyzed. The relative error rates of the four prediction methods are compared, demonstrating an improvement in the operation efficiency prediction accuracy of typical work types under high-altitude alpine conditions.

\section{Analysis of Key Factors Affecting the Efficiency of Typical Work Types}

Air drilling is widely employed in the study of humanmachine efficiency due to its high labor intensity, harsh operating environment, high requirements for humanmachine linkage operation, and outstanding safety issues. Therefore, we selected air drilling as the research object to explore the variations in its operating efficiency under highaltitude alpine conditions. Figure 1 presents an actual scene of the air drill job site.

2.1. Establishment of an Evaluation Index System for Air Drilling Efficiency. This study adopts the theory of ergonomics through the collaborative relationship between operators, operating machinery, and operating environment to analyze the factors affecting the pneumatic drilling efficiency in high-altitude mining areas from the perspectives of personnel, equipment, and environment. Based on the results, we develop a hierarchical structure of the air drilling efficiency evaluation system. Table 1 reports the index system.

2.2. Key Influencing Factors of Mining. Considering the fuzzy extension among the indicators of different mine levels and the distinct order of magnitude and information content among the data, the entropy weight method was adopted to weight each index. This ensures the objectivity of weighting and avoids the influence of experience bias on the results. The entropy method essentially determines the weight based on the information entropy differences contained in each element. The greater the difference between an index value in the evaluation object, the more effective the information and the greater the weight assigned [29, 30].

Furthermore, in order to comprehensively and systematically analyze the coupling and correlation between multiple efficiency influencing factors and the overlapping effects of data at different altitudes, we employ PCA to screen key indicators [31-33]. This allows us to simplify the evaluation. The theoretical model is described as follows.

2.2.1. Indicator Standardization. In order to eliminate the influence caused by the differences in magnitude and dimension of each index, we perform standardization via equations (1)-(3):

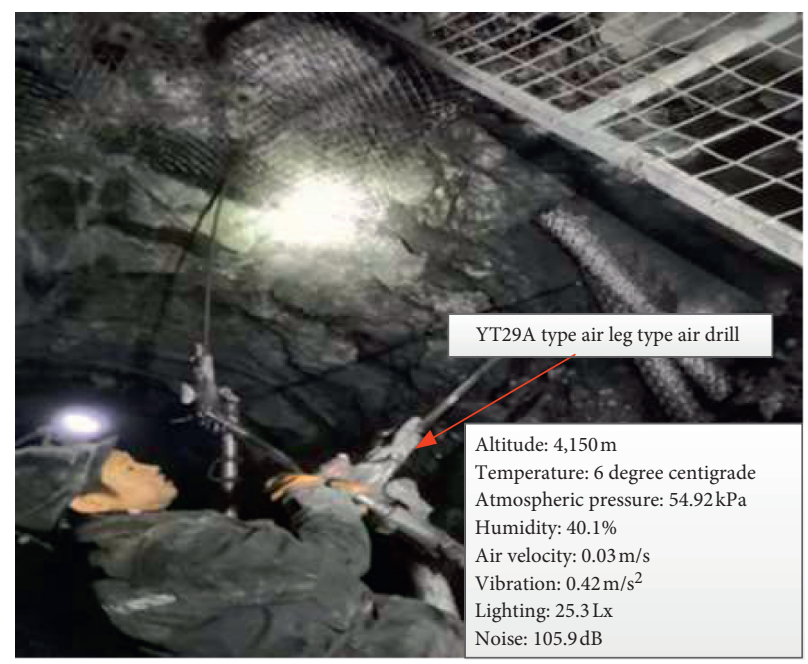

FIgURE 1: Air drill operation.

$$
\begin{aligned}
& Z_{i j}=\frac{X_{i j}-\overline{X_{j}}}{S_{j}}, \\
& \overline{X_{j}}=\frac{\sum_{j=0}^{n} X_{i j}}{n}, \\
& S_{j}^{2}=\frac{\sum_{j=0}^{n}\left(X_{i j}-\overline{X_{j}}\right)^{2}}{n-1},
\end{aligned}
$$

where $i=1,2, \ldots, n$ and $j=1,2, \ldots, P$, and $i$ and $p$ correspond to the number of samples and the number of indicators, respectively.

2.2.2. Indicator Weight. The entropy weight method is adopted to calculate the importance weight of each index $W=\left\{W_{1}, W_{2}, \ldots, W_{n}\right\}$. The entropy value of each index is derived via equation:

$$
E_{j}=\frac{\sum_{i=1}^{m} P_{i j} \operatorname{Ln} P_{i j}}{\operatorname{Ln} m},
$$

for $P_{i j}=0$ and $P_{i j} \operatorname{Ln} P_{i j}=0$.

The weight of each index $W_{j}$ is subsequently calculated as follows:

$$
\begin{aligned}
W_{j} & =\frac{1-E_{j}}{\sum_{j=1}^{n}\left(1-E_{j}\right)}, \\
\sum_{j=1}^{n} W_{j} & =1 .
\end{aligned}
$$

The columns of the data matrix determined from step one are multiplied by weight $W_{j}$ to obtain the following:

$$
Z_{i j}^{\prime}=W_{j} Z_{i j}, \quad i=1, \ldots, m ; j=1, \ldots, n .
$$


TABLE 1: Index system of the factors influencing the working efficiency of pneumatic drills in high-altitude mining areas.

\begin{tabular}{|c|c|c|}
\hline First-level indexes & Second-level indexes & Third-level indexes \\
\hline Air drill operation efficiency in high-altitude mining areas & Equipment factors & $\begin{array}{c}\text { Age } \\
\text { Operating years } \\
\text { Education level } \\
\text { Physical and mental fatigue } \\
\text { Degree of acclimation } \\
\text { Output power of diesel engine } \\
\text { Load and energy consumption of air drilling rig } \\
\text { Aging and wear rate of air drilling rig } \\
\text { Temperature } \\
\text { Humidity } \\
\text { Atmospheric pressure } \\
\text { Air velocity } \\
\text { Vibration } \\
\text { Lighting } \\
\text { Noise }\end{array}$ \\
\hline
\end{tabular}

2.2.3. Correlation Matrix of the Normalized Data. Equations (7) and (8) are used to calculate the correlation matrix:

$$
\begin{aligned}
R & =\left(r_{j k}\right)_{p \times p}, \quad K=1,2, \ldots, P, \\
r_{j k} & =\operatorname{COV}\left(X_{j}-X_{k}\right) .
\end{aligned}
$$

2.2.4. Eigenvalues and Eigenvectors. The eigenvalues of matrix $R, \lambda_{1}, \lambda_{2}, \ldots, \lambda_{p}$, and the corresponding feature vector $L_{j}=a_{1 j}, a_{2 j}, \ldots, a_{p j}$ are determined. Following this, the variance contribution rate of the Kth principal component is derived via equation:

$$
\alpha_{k}=\frac{\lambda_{k}}{\sum_{i=1}^{p} \lambda_{i}},
$$

while the cumulative contribution rate of the principal components is calculated using equation:

$$
\alpha_{\text {sum }}=\frac{\sum_{i=1}^{m} \lambda_{i}}{\sum_{i=1}^{p} \lambda_{i}} .
$$

As the loss data contains the minimum amount of information, the number of principal components should be minimized (typically to $m$ ) to simplify the calculation, and the cumulative contribution rate of the variance should be greater than or equal to $85 \%$.

2.2.5. Comprehensive Evaluation of Selected Principal Components. Equation (11) is employed to determine the principal components of the sample. The selected $m$ principal components are subsequently calculated and sorted via equation (12) to obtain the index evaluation results:

$$
\begin{aligned}
M_{i j} & =Z_{n, p} \times\left[l_{1}, l_{2}, \ldots, l_{p}\right], \\
F_{i} & =\sum_{j=1}^{m} \alpha_{j} M_{i, j} .
\end{aligned}
$$

\section{Establishment of the Prediction Model for the Operational Efficiency of High-Altitude Air Drilling}

The proposed SeqGAN generative adversarial network aims to overcome the difficulties in obtaining experimental data and the limited amount of sample data. GAN has been proved to have a strong anti-interference ability in practical applications and has become a research hotspot in the field of deep learning in recent years. In addition, SeqGaN networks can effectively expand and increase the robustness of sparse and discrete data. Integrating $X$ with a genetic algorithm achieves global optimization can overcome the tendency of neural networks to fall into local optimums. This is fused with the BP neural network optimized via the improved multiobjective genetic algorithm to form the SeqGAN-GABP mixed algorithm. The proposed framework initially employs the SeqGAN generative adversarial network to expand and increase the robustness of the discrete sample data. The expanded sample data is then input into the GA-BP network for training. Figure 2 presents the algorithm flow.

3.1. SeqGAN Network. Previous research has proved the strong anti-interference ability of the generative adversarial network (GAN) in practical applications [34, 35]. In the current study, GAN is used to solve the deep neural network training problem. In particular, we divide the GAN network into two functional modules: the generator $(G)$ and discriminator $(D)$. Figure 3 displays the network structure.

We assume that generator $G$ samples random noise $z$ from an arbitrary distribution as the input, and an accurate fit is achieved between the generator $G$ and the distribution of the real data. True data $x$ and data $G(z)$ generated by the generator are entered into discriminator $D$. During training, the discriminator $D$ is instructed to perform its best attempt to distinguish whether the sample is real data $X$ or data $G(Z)$ generated by the generator. The generator and discriminator perform a game confrontation update iteration until the Nash equilibrium is reached and the generated data $G(z)$ is 


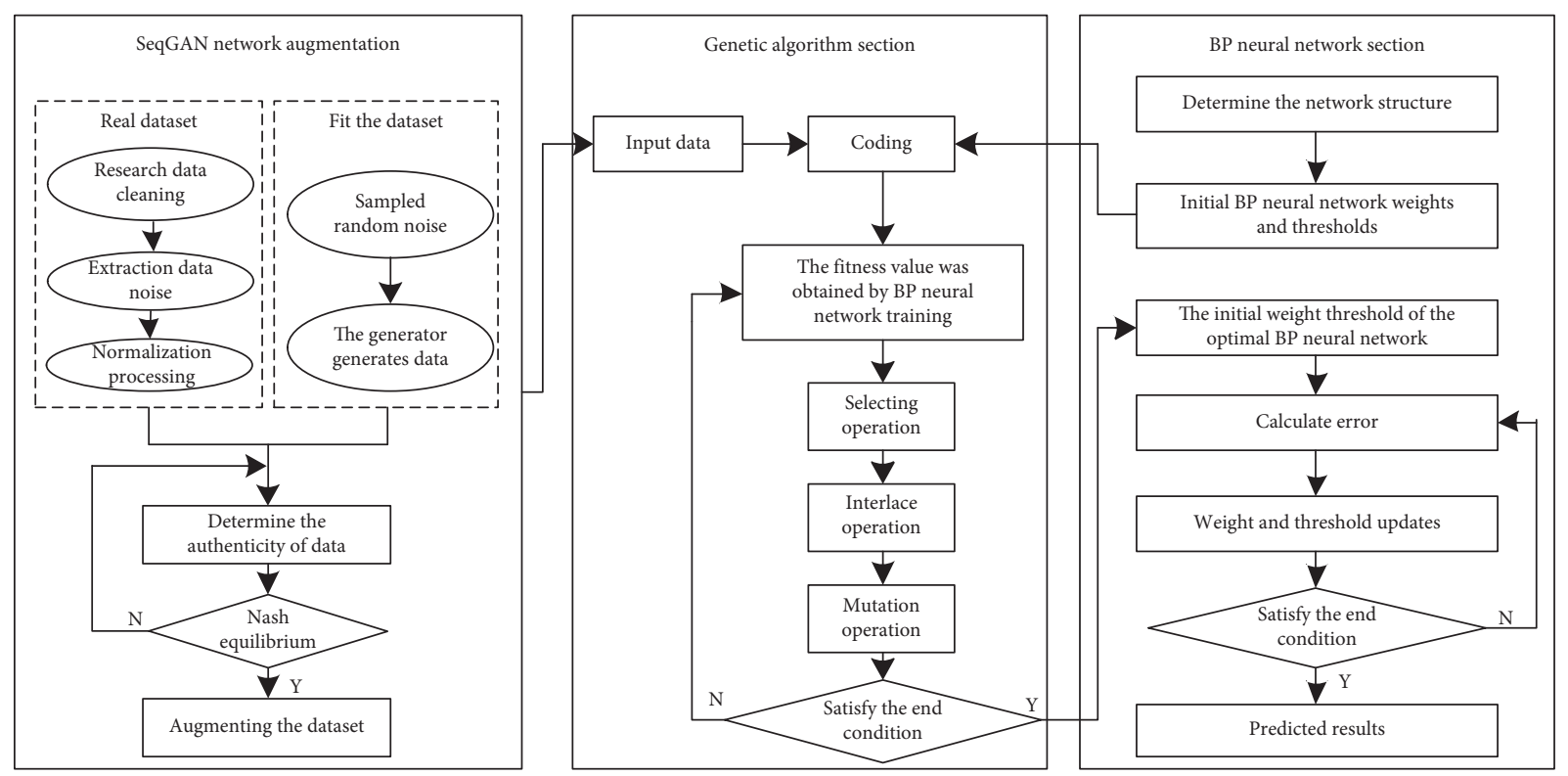

Figure 2: SeqGAN-GABP mixed algorithm flowchart.

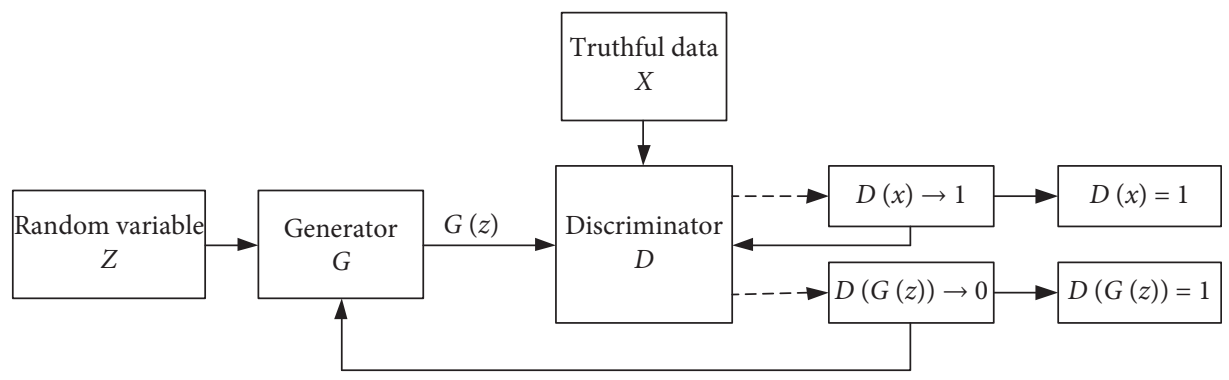

FIGURE 3: Generative adversarial network structure diagram.

considered to be extremely close to real data $x$. Equation (13) describes the training process for GAN:

$$
\min _{G} \max _{D} E_{x \sim P \text { date }} \log [D(x)]+E_{z \sim P z} \log [1-D(G(z))],
$$

where $P$ date represents the distribution of the real data and $P z$ is the noise data, which can obey any distribution. As the sample data is sparse and discrete, the discrete output of the generator in the model cannot easily transfer the gradient update from the discriminator to the generator. Therefore, based on the GAN network, we select the random LSTM (long-short-term memory) distribution and sample this distribution to obtain a positive sample. Following this, we perform the Monte Carlo Tree Search (MCTS) via weighted sampling at the first node. SeqGAN is then employed to expand the sample data.
Figure 4 presents a schematic diagram of SeqGAN, where the existing red dot denotes the present state and the subsequent red dot denotes the action. The generator determines a sequence to maximize the expectation of the feedback value, such as that described in equation:

$$
J(\theta)=E\left[R_{T} \downarrow s_{0 . \theta}\right]=\sum_{y 1 \in Y} G_{\theta}\left(y_{1} \downarrow s o\right) \cdot Q_{D_{\varnothing}}^{G_{\theta}}\left(s_{0}, y_{1}\right),
$$

where $J(\theta)$ is the expectation of the feedback value, $G_{\theta}$ is the generator model, and $Q$ is the return value of the discriminator, which can be expressed as

$$
Q_{D_{\varnothing}}^{G_{\theta}}\left(a=y_{T}, s=Y_{1: T-1}\right)=D_{f}\left(Y_{1: T}\right)
$$

MCTS is then employed to complete the content after $y_{t}$, and the average is determined: 


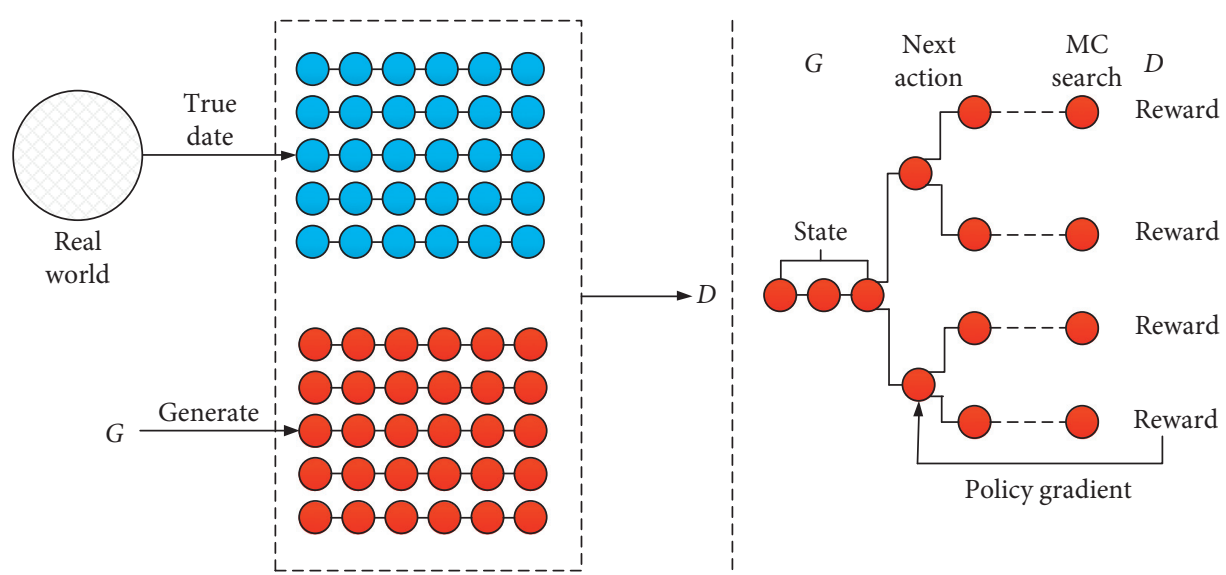

Figure 4: Schematic diagram of SeqGAN.

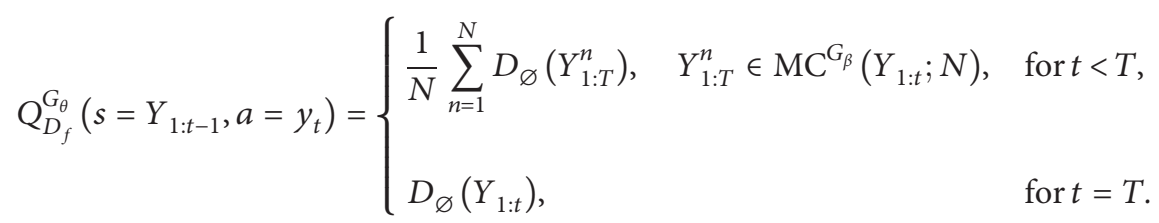

Following the generation of realistic sequences, equation (17) is implemented to train the discriminator:

$$
\min _{\varnothing}-E_{Y \sim P \text { date }}\left[\log D_{\varnothing}(Y)\right]-E_{Y \sim G_{\theta}}\left[\log \left(1-D_{\varnothing}(Y)\right)\right] .
$$

The updated discriminator is used to update the discrimination. This update can be performed via a gradient descent, such as that described in equations (18) and (19):

$$
\begin{gathered}
\theta \leftarrow \theta+a_{h} \nabla_{\theta} J(\theta), \\
\nabla_{\theta} J(\theta)=E Y_{1: t-1} \sim G_{\theta}\left[\sum_{y_{t} \in Y} \nabla_{\theta} G_{\theta}\left(y_{t} \mid Y_{1: t-1}\right) \cdot Q_{D_{\varnothing}}^{G_{\theta}}\left(Y_{1: t-1}, y_{t}\right)\right],
\end{gathered}
$$

where $a_{h}$ represents the learning rate.

3.2. GA-BP Neural Network. The BP neural network is a widely used multilayer feedforward neural network. The training of this network typically relies on the error back propagation. More specifically, the weight and threshold of the network are adjusted via the backpropagation, allowing for numerous nonlinear problems to be solved. Thus, we establish the GA-BP neural network model to predict pneumatic drilling efficiency [36, 37].

The GA-BP algorithm is divided into three components: (1) the establishment of the BP neural network model, (2) the optimization of the BP network via the genetic algorithm, and (3) the prediction of the optimized BP neural network. In the current study, we set the training error to the chromosome fitness value and calculate $F$ as follows:

$$
F=K\left(\sum_{i=1}^{n} a b s\left(y_{i}-o_{i}\right)\right)
$$

where $n$ is the number of output nodes, the actual result of the $i$ th node, the prediction result of the $i$ th node, followed by the selection, crossover, and mutation operations.

The main parameters of genetic algorithm are population size, crossover probability, mutation probability, evolutionary algebra, and population initialization method. These parameters determine whether a population will mature early. If the population size is too small, inbreeding will lead to the occurrence of pathological genes, while if the population size is too large, the efficiency of the algorithm will decline, so the common population size between 100 and 200 is appropriate. The value is generally set between 0.4 and 0.99 . When the mutation probability is too large, the good genes will also be destroyed, and the good genes from the father generation cannot be effectively passed to the offspring. When the population diversity decreases, it is easy to fall into the local optimum, which is generally between 0.0001 and 0.2 .

3.2.1. Operation Selection. We employ the roulette method (equations (21) and (22)) to select the operation due to its tendency to select chromosomes with excellent fitness values:

$$
\begin{gathered}
f_{i}=\frac{k}{F_{i}}, \\
P_{i}=\frac{f_{i}}{\sum_{i=1}^{N} f_{i}},
\end{gathered}
$$

where $F_{i}$ is the fitness value of individual $i, K$ is the coefficient, and $N$ is the total number of chromosomes. 
3.2.2. Interlace Operation. Cross processing requires a pair of chromosomes to be determined in an individual with a random crossing position, described as

$$
\left\{\begin{array}{l}
a_{m j}=a_{m j}(1-b)+a_{i j} b \\
a_{i j}=a_{i j}(1-b)+a_{m j} b
\end{array}\right.
$$

where $b$ is a random number between $[0,1]$.

3.2.3. Mutation Operation. The mutation operation requires the random mutation of the $y$ gene $a_{x y}$ on the $x$ chromosome based on a predefined probability:

$$
a_{x y}= \begin{cases}a_{x y}+\left(a_{x y}-a_{\max }\right) * f(g), & r>0.5, \\ a_{x y}+\left(a_{\min }-a_{x y}\right) * f(g), & r \leq 0.5,\end{cases}
$$

where max and min are the upper and lower bounds of the chromosome genes, respectively, $g$ is the number of iterations, and $r$ is a random number between intervals $[0,1]$. The optimal initial weights and thresholds processed via the genetic algorithm are substituted into the $\mathrm{BP}$ neural network for predictions.

\section{Experiment}

4.1. Selection of Experimental Sites. We selected three highaltitude metal mines in Xinjiang as the research objects. Table 2 lists the details of each mine.
Figure 5 presents a satellite image of the mine topography.

Raw data was collected from April to November 2019 via field measurements, theoretical analysis, and questionnaires.

4.2. Experimental Data Acquisition. Due to the strong subjective perception of operators, personnel index data was collected using a combination of online and offline questionnaires. A total of 162 questionnaires were distributed to the three mines, 151 of which were returned.

The equipment index data employed in the current study were taken from previous literature due to the difficulties in handling data acquisition devices. The output power of diesel engines is calculated as follows [38]:

$$
\eta=\frac{N_{e}}{N_{i}}=\frac{N_{e}}{N_{e}+N_{m}},
$$

where $\eta$ is the diesel engine output power, $N_{i}$ is the indicated power of the internal combustion engine, $\mathrm{kW}, N_{e}$ is the effective power of the internal combustion engine, $\mathrm{kW}$, and $N_{m}$ is the internal combustion engine mechanical loss power, $\mathrm{kW}$, and is determined using the following equation:

$$
N_{m}=\left[0.00697(\varepsilon-4)+0.0483 \times 10^{-3} n+0.0401 \times 10^{-2} V_{m}^{2}\right] \frac{V_{h} n i}{30 \tau} \times 10^{-3}
$$

where $\varepsilon$ is the compression ratio, $V_{m}$ is the average speed of the piston, $\mathrm{m} / \mathrm{s}, V_{h}$ is the working volume of the cylinder, $L$, $\tau$ is the number of strokes, $n$ is the rotational speed, $r / \mathrm{min}$, and $i$ is the number of cylinders. $N_{e}$ in equation (25) can be described as

$$
N_{e}=\frac{n_{e} n_{v} H_{u} P_{a} V_{h} n i}{30 \operatorname{Ral}_{0} T_{a} \tau},
$$

where $n_{e}$ is the effective thermal efficiency, $n_{V}$ is the charging efficiency, $n_{V}=\left(V_{a} / V_{h}\right), V_{a}$ is the air intake volume in the air inlet state, and $H_{u}$ is the low calorific value of the fuel.

Table 3 provides details of the environmental indicator information collected using field measurements.

\subsection{Calculation of Key Factors Influencing Operational} Efficiency. Following the reduction in dimensions of the original data via the PCA and fusion entropy weight method, the following five key efficiency influencing factors were determined: the mechanical efficiency of the diesel engine and the age, atmospheric pressure, acclimation degree, and load and energy consumption of the pneumatic drill $\left(x_{1}-x_{5}\right.$, respectively). The outliers and missing values were eliminated to yield 20 groups of effective data that were then used as the evaluation factors. Note that two decimal values were retained for the experimental data. Table 4 reports the original data.

4.4. SeqGaN-GABP Algorithm Steps. The proposed SeqGaNGABP algorithm can be divided into the following five steps.

(1) A sequence dataset is determined consisting of the 20 real data structures. Generator $G_{\theta}$ is trained with parameter $\theta$ to generate sequence $y_{1: T}=\left(y_{1}, y_{2}, \ldots, y_{t}, \ldots, y_{T}\right), y_{t} \in Y$, and discriminator $D_{\varnothing}$ is trained with parameter $\varnothing$ to improve generator $G_{\theta}$.

(2) The LSTM model is introduced to provide training datasets. More specifically, a randomly initialized Oracle model is employed to generate the real data distribution $P\left(x_{t} \mid x_{1}, \ldots, x_{t-1}\right)$. The normal distribution $N(0,1)$ is taken as the distribution $G_{\text {oracle }}\left(x_{t} \mid x_{1}, \ldots, x_{t-1}\right)$ of the real data described by the Oracle model to initialize the LSTM parameters. LSTM is then used to generate model training set $S$.

(3) The trained model is implemented to expand the 20sample dataset, resulting in a 300-sample dataset for the GA-BP neural network prediction. 
TABLE 2: Basic information of mining area.

\begin{tabular}{|c|c|c|c|c|}
\hline Mine & $\begin{array}{l}\text { Type of produced } \\
\text { metal }\end{array}$ & $\begin{array}{l}\text { Lowest altitude } \\
(\mathrm{m})\end{array}$ & $\begin{array}{l}\text { Highest altitude } \\
(\mathrm{m})\end{array}$ & $\begin{array}{l}\text { Average altitude } \\
\qquad(\mathrm{m})\end{array}$ \\
\hline Beizhan mining in Hejing county & Iron & 3,160 & 4,575 & 3,800 \\
\hline Baiyuanfeng mining in Aktao county & Manganese & 3,780 & 4,365 & 4,100 \\
\hline $\begin{array}{l}\text { Dahongshan mining area of Aktash iron mine, Wuqia } \\
\text { county }\end{array}$ & Iron & 3,077 & 3,239 & 3,160 \\
\hline
\end{tabular}

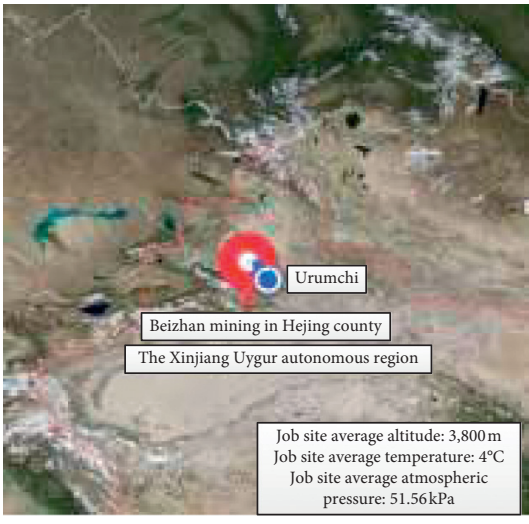

(a)

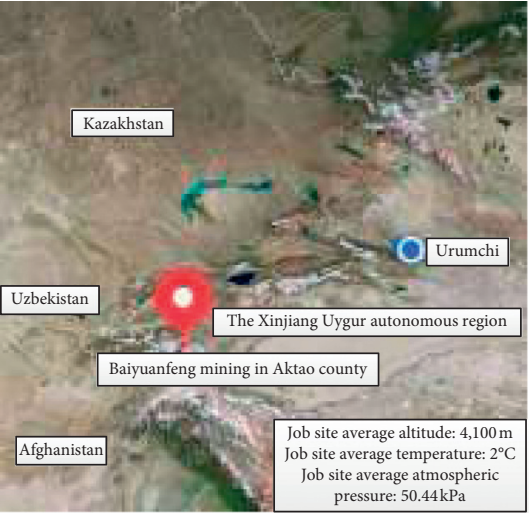

(b)

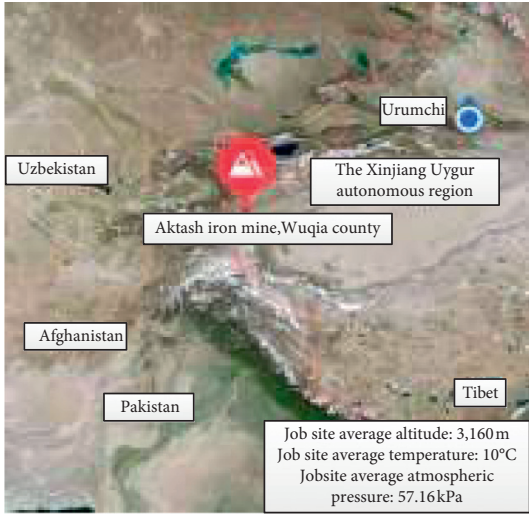

(c)

FIGURE 5: Satellite image of each of the three experimental sites. (a) Beizhan mine. (b) Baiyuanfeng mine. (c) Aktash iron mine.

TABLE 3: Measuring parameters and instruments.

\begin{tabular}{lcc}
\hline Measurement parameters & Instrument & Technical parameters \\
\hline Temperature & STT-T temperature sensor $(\mathrm{Pt} 100)$ & Range: $-50-120^{\circ} \mathrm{C}$ precision: $\pm 0.15^{\circ} \mathrm{C}$ \\
Humidity & STH-TW2 humidity transmitter & Humidity range: $0-100 \% \mathrm{RH}$, precision: $\pm 2 \% \mathrm{RH}$ \\
Atmospheric pressure & $\mathrm{J} 1012$ barometer & Range: $80-106 \mathrm{kPa}, \mathrm{precision}: \pm 0.25 \mathrm{kPa}$ \\
Air velocity & YGC-FS air velocity transducer & Range: $0-45 \mathrm{~m} / \mathrm{s}$, resolution: $0.1 \mathrm{~m} / \mathrm{s}$ \\
Vibration & GM63A vibration meter & Accuracy: $\pm(0.3+0.03 \mathrm{~V}) \mathrm{m} / \mathrm{s}(\mathrm{V}$ means air speed) \\
Lighting & RS485 & Range: $0.1-199.9 \mathrm{~m}^{2} / \mathrm{s}$, precision: $\pm 5 \% \mathrm{~Hz}$ \\
Noise & TES1352S & Range: $0-65535 \mathrm{Lux}$, precision: $\pm 7 \%$ \\
\hline
\end{tabular}

TABLE 4: Original survey data sheet.

\begin{tabular}{lcccccc}
\hline $\begin{array}{l}\text { Numerical } \\
\text { order }\end{array}$ & $\begin{array}{c}x_{1} \\
(\%)\end{array}$ & $\begin{array}{c}x_{2} \\
(\text { age })\end{array}$ & $\begin{array}{c}x_{3} \\
(\mathrm{kPa})\end{array}$ & $\begin{array}{c}x_{4} \\
(\text { rank })\end{array}$ & $\begin{array}{c}x_{5} \\
(\%)\end{array}$ & $Y(\%)$ \\
\hline 1 & 69.65 & 36 & 58.28 & 8.2 & 85.62 & 83.34 \\
2 & 64.78 & 46 & 51.56 & 4.95 & 83.78 & 44.46 \\
3 & 66.42 & 44 & 53.8 & 6.03 & 84.38 & 55.56 \\
4 & 67.17 & 34 & 54.92 & 6.48 & 84.71 & 66.67 \\
5 & 68.07 & 38 & 56.04 & 6.97 & 84.96 & 72.21 \\
6 & 69.62 & 48 & 58.28 & 7.96 & 85.62 & 83.36 \\
7 & 68.76 & 42 & 57.16 & 7.52 & 85.33 & 77.78 \\
8 & 66.39 & 28 & 53.8 & 6.03 & 84.38 & 55.54 \\
9 & 65.58 & 38 & 52.68 & 5.48 & 84.12 & 50.06 \\
10 & 64.04 & 42 & 50.44 & 4.05 & 83.48 & 38.38 \\
11 & 64.76 & 37 & 51.56 & 4.96 & 83.81 & 44.43 \\
12 & 67.22 & 44 & 51.56 & 6.48 & 84.68 & 66.65 \\
13 & 68.78 & 49 & 57.16 & 7.52 & 85.27 & 77.76 \\
14 & 66.42 & 34 & 56.04 & 6.03 & 84.43 & 55.56 \\
15 & 66.38 & 37 & 57.16 & 6.19 & 84.38 & 55.52 \\
16 & 68.03 & 45 & 53.8 & 7.13 & 84.97 & 72.21 \\
17 & 67.23 & 42 & 54.92 & 6.51 & 84.72 & 66.67 \\
18 & 65.58 & 36 & 52.68 & 5.58 & 84.09 & 49.98 \\
19 & 67.18 & 39 & 54.92 & 6.52 & 84.68 & 66.68 \\
20 & 67.96 & 41 & 56.04 & 7.04 & 85.08 & 72.22 \\
\hline
\end{tabular}

(4) The input layer of the GA-BP neural network is set as the following five neurons: mechanical efficiency, age, atmospheric pressure, acclimation degree of the diesel engine, and load and energy consumption of the pneumatic drill. The output layer is the operation efficiency of the wind rig and the number of hidden layer nodes is determined as follows:

$$
N=\sqrt{A+B}+C
$$

where $A$ is the number of output neurons, $B$ is the number of input neurons, and $C$ is a constant between $[1,10]$. In our work, the output error is minimized for $N=9$, and thus, we select 9 neurons in the hidden layer.

(5) After the topology of the neural network is determined, the target error is defined and the training begins. The training ends when the target error is satisfied. The number of iterations, target error, and learning efficiency are set as 1,000, 0.0001, and 0.01, respectively. The iterations stop when either the 
TABLE 5: Multiple linear regression analysis results.

\begin{tabular}{|c|c|c|c|}
\hline Model & Coefficient & Residual error & Colinear statistics \\
\hline Constant quantity & -1483.363 & \multirow{6}{*}{2.164} & \\
\hline Output power of diesel engine & 6.363 & & 8.432 \\
\hline Age & 0.08 & & 1.834 \\
\hline Oxygen content & 10.381 & & 6.483 \\
\hline Degree of acclimation & -6.902 & & 1.936 \\
\hline Load and energy consumption of air drilling rig & 12.189 & & 6.914 \\
\hline
\end{tabular}

TABLE 6: Comparison of prediction results of air drill operation efficiency.

\begin{tabular}{lcccccccccc}
\hline Numerical order & Altitude $(\mathrm{m})$ & $W(\%)$ & $W_{1}(\%)$ & $\Delta_{1}(\%)$ & $W_{2}(\%)$ & $\Delta_{2}(\%)$ & $W_{3}(\%)$ & $\Delta_{3}(\%)$ & $W_{4}(\%)$ & $\Delta_{4}(\%)$ \\
\hline 6 & 3,860 & 83.36 & 84.12 & 0.91 & 82.43 & 1.13 & 81.38 & 2.43 & 87.42 & 4.87 \\
13 & 3,920 & 77.76 & 77.91 & 0.19 & 78.17 & 0.52 & 78.65 & 1.14 & 78.74 & 1.26 \\
16 & 3,990 & 72.21 & 72.18 & 0.04 & 72.46 & 0.35 & 72.86 & 0.9 & 73.1 & 1.23 \\
19 & 4,080 & 66.68 & 65.49 & 1.81 & 64.64 & 3.15 & 64.49 & 3.4 & 64.36 & 3.6 \\
\hline
\end{tabular}

setting accuracy is satisfied or the maximum number of iterations is reached.

4.5. Multiple Linear Regression Mathematical Algorithm. We compare the SeqGAN-GABP with the multiple linear regression model due to its ability to establish quantitative functional relationships $[39,40]$. The experimental data in Table 2 was analyzed and a mathematical model was built via IBM SPSS Statistics 25 to estimate the unknown model parameters. The regression model was used to predict variations in the dependent variables (Table 5).

We determined a residual value of 2.164 , which is close to the standard of 2 . This indicates that the residual is independent and conforms to the establishment of the multiple linear regression model. In addition, the collinearity statistics are all less than 10, suggesting the absence of multicollinearity. More specifically, there is no linear relationship between the independent variables. For high-altitude alpine conditions, the relationship between the working efficiency of the pneumatic drill and the mechanical efficiency, age, atmospheric pressure, acclimation degree, and air drilling rig load and energy consumption of the of the diesel engine is determined as

$$
\begin{aligned}
y= & -1483.363+6.363 x_{1}+0.08 x_{2}+10.381 x_{3}-6.902 x_{4} \\
& +12.189 x_{5} .
\end{aligned}
$$

The results indicate that, for altitudes between $3,800-4,500 \mathrm{~m}$, the mechanical efficiency of the diesel engine and the working efficiency of pneumatic drilling increase by $1 \%$ and $6.363 \%$, respectively. For each unit increase in the wind driller age, the efficiency is enhanced by $0.08 \%$. Moreover, the wind driller efficiency increases by $10.381 \%$ for each unit increase in atmospheric pressure. The pneumatic drill efficiency decreases by $6.902 \%$ for each unit increase of learning degree, and when the load and energy consumption of the wind drill increase by $1 \%$, the working efficiency increases by $12.189 \%$.

4.6. Comparison of Operational Efficiency Forecast Results of the Pneumatic Drill. In order to test the prediction accuracy of the SeqGAN-GABP hybrid algorithm model, we compare it with the GA-BP neural network, BP neural network, and multiple linear regression analysis method using the No. 6, No. 13, No. 16, and No. 19 datasets collected from Aketashi Iron Mine in Wuqia County, Xinjiang (No. 3, $839-4,077 \mathrm{~m}$ of $\mathrm{I}-1$ ore body in the Dahongshan mining area).

Table 6 reports the results, where $W$ is the measured value of the pneumatic drilling operation efficiency, $W_{1}, W_{2}, W_{3}$, and $W_{4}$ are the predicted values, and $\Delta_{1}, \Delta_{2}$, $\Delta_{3}$, and $\Delta_{4}$ are the relative errors between the measured and predicted values of the SeqGAN-GABP hybrid algorithm, the GA-BP neural network, the BP neural network, and the multiple linear regression analysis, respectively.

Figure 6 compares the measured and predicted values of the pneumatic drill working efficiency in high-altitude alpine regions. The neural network method surpasses the multiple linear regression analysis in terms of accurately predicting the pneumatic drilling efficiency. The proposed model can better predict the working efficiency of pneumatic drilling under high-altitude alpine conditions compared to the other three models.

Figure 7 presents the relative error trend of the four prediction models, all of which exhibit maximum relative errors less than 5\%. This indicates that there are no significant differences between the experimental and predicted values of the models. Furthermore, the overall fluctuations of the four prediction methods are not too high, highlighting the stability of each model. 


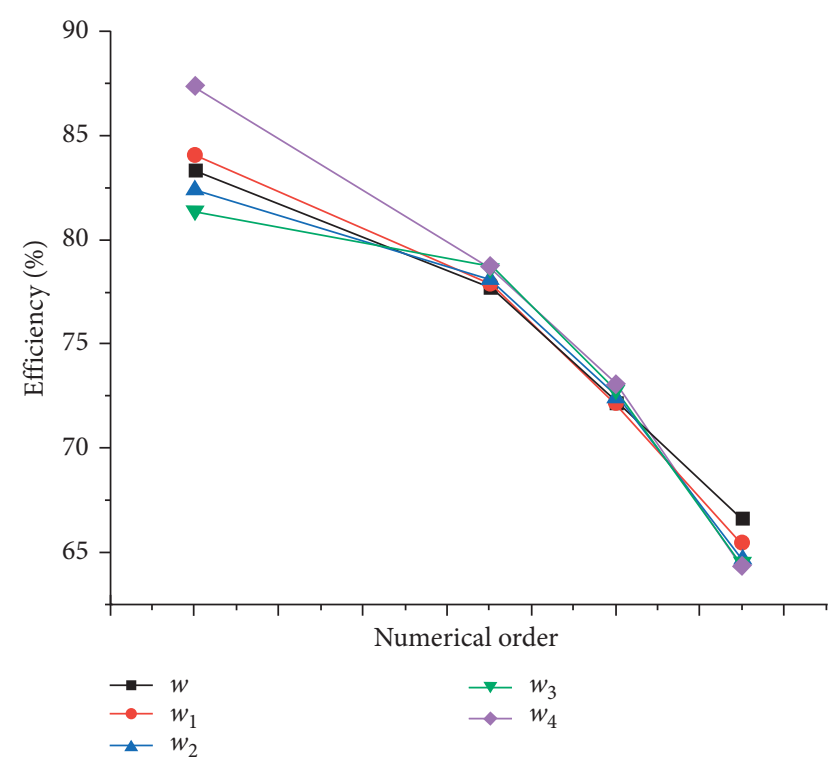

Figure 6: Comparison of the air drill efficiencies determined from the four models.

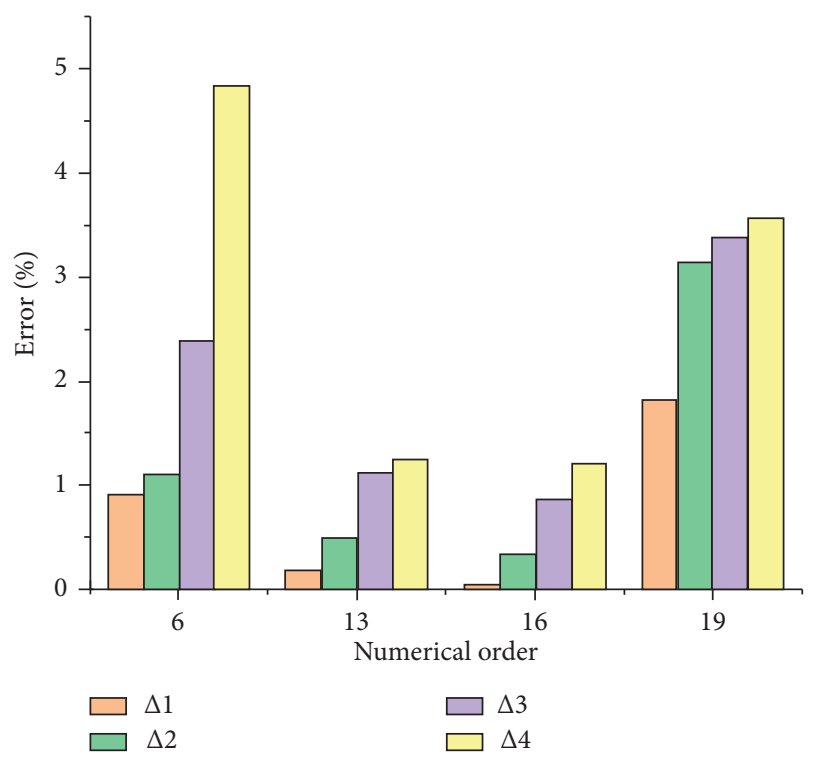

FIgURE 7: Relative error of the four forecasting methods.

\section{Conclusion}

In the current study, we established an evaluation index system of the pneumatic drilling working efficiency with a three-layer structure and 15 influencing factors. The proposed framework combines existing research with the environmental characteristics of high-altitude alpine mining areas. We applied ergonomics theory to evaluate the collaborative relationship between operators, working machinery, working environment, and unique and mutually restricted design variables. We focused on objectively analyzing the coupling correlation, multiple influencing factors, and overlapping influence of data under high-altitude and alpine conditions. In particular, we employed PCA and the fusion entropy weight method to screen the key indicators and subsequently selected five key efficiency influencing factors. We optimized the BP neural network via a multiobjective genetic algorithm by combining the SeqGAN generative adversarial network to form the SeqGAN-GABP mixed algorithm. The proposed algorithm expands the sample size and does not tend to fall into a local optimum, thus increasing the accuracy of the prediction network results.

The air drilling operation efficiency at three high-altitude metal mines in Xinjiang was subsequently predicted and verified. The GA-BP neural network, BP neural network, and multiple linear regression analysis method were compared with the proposed model.

The results demonstrate the ability of the proposed model to effectively predict the working efficiency of typical work types under high-altitude alpine conditions. The model achieved the desired goal and can act as an effective evaluation and assessment tool for the management of highaltitude mining areas and can also provide support for the safe production and organizational optimization of highaltitude alpine mines. Future research will focus on applying the mixed prediction model to other tasks.

We apply the theory of ergonomics to practical engineering problems of high-altitude alpine metal mines via field investigations and theoretical calculations. In addition, the working efficiency of typical work types in high-altitude alpine metal mines is investigated from the perspective of the overall synergistic relationship between human and machine environments. Improving the efficiency of the model is reserved for future research.

\section{Data Availability}

The data used to support the findings of the study are included in supplementary information files.

\section{Conflicts of Interest}

The authors declare no conflicts of interest regarding the publication of this paper.

\section{Acknowledgments}

This research was funded by the National Key R\&D Program of China "Man-Machine Efficacy and Emergency Rescue Technology in High-Altitude Alpine Mining” (no. 2018YFC0808406).

\section{Supplementary Materials}

The field survey raw data are provided in the supplementary information files. (Supplementary Materials)

\section{References}

[1] Z. Chen, H. Nailian, and D. Shaofu, "Research on evaluation system of green mines in non-ferrous metal industry," Mining R\&D, vol. 39, pp. 150-155, 2019. 
[2] N. Xingxin, Z. Shudu, and F. Shanshan, "Simulation probe on the locally super-charged air curtain control at the high altitude mine heading face," Journal of Safety and Environment, vol. 20, pp. 127-135, 2020.

[3] L. Guoqing, Z. Ya-ming, and G. Jian, "Study on pressurization ventilation in plateau mine," Metal Mine, vol. 2, pp. 151-156, 2017.

[4] L. Changhong, X. Yong-gang, and W. Yu, "Review and prospects for understanding deformation and failure of rock slopes in cold regions with high altitude," Chinese Journal of Engineering, vol. 41, pp. 1374-1386, 2019.

[5] L. Chaoqiang, H. Wenjun, and L. Hao, "Quantified self: construction of big data analysis platform for human efficacy evaluation of complex information system," Journal of CAEIT, vol. 12, pp. 563-569, 2017.

[6] A. Egger, M. Niederer, K. Tscherny et al., "Influence of physical strain at high altitude on the quality of cardiopulmonary resuscitation," Scandinavian Journal of Trauma, Resuscitation and Emergency Medicine, vol. 28, no. 1, p. 19, 2020.

[7] C. Qiulin, L. Guoli, and L. Yuia, "Study on unsafe behavior management of workers in Dongguashan copper mine," Mining Technology, vol. 15, pp. 67-69, 2015.

[8] G. Anamika, Pooja, M. Sharma et al., "Intermittent normobaric hypoxia facilitates high altitude acclimatization by curtailing hypoxia-induced inflammation and dyslipidemia," European Journal of Physiology, vol. 471, pp. 949-959, 2019.

[9] W. Qiujun, Y. Li, and W. Feng, "Study of construction control technology for super-long high altitude highway tunnel in low pressure and low oxygen environment: technical standards for oxygen supply, key ventilation technology, construction personnel organization and construction equipment efficiency," Tunnel Construction, vol. 37, pp. 973-979, 2017.

[10] Z. Xin, G. Chun, and W. Xin, "Evaluation on labor intensity of workers in Guigala high-altitude tunnel," Tunnel Construction, vol. 39, pp. 1141-1146, 2019.

[11] A. Reza, Z. Vahid, and A. Ziba, "Characteristics of headache at altitude among trekkers: a comparison between acute mountain sickness and non-acute mountain sickness headache," Asian Journal of Sports Medicine, vol. 3, pp. 126-130, 2012.

[12] D. Yang, L. a. Cao, Y. Liu, and Z. Zhang, "Experimental and numerical investigation on a novel gas turbocharging system for diesel engine power recovery at high altitude," Journal of Mechanical Science and Technology, vol. 33, no. 10, pp. 5061-5072, 2019.

[13] M. Blancher, M. Repellin, M. Maignan et al., "Accuracy of low-weight versus standard syringe infusion pump devices depending on altitude," Scandinavian Journal of Trauma, Resuscitation and Emergency Medicine, vol. 27, no. 1, p. 65, 2019.

[14] H. Yucheng and W. Yumin, "Influence of high altitude on performance of mine axial flow fan and reasonable selection of fan," China Mining Magazine, vol. 1, pp. 133-136, 2020.

[15] J. E. Davis, D. R. Wagner, N. Garvin, D. Moilanen, J. Thorington, and C. Schall, "Cognitive and psychomotor responses to high-altitude exposure in sea level and highaltitude residents of Ecuador," Journal of Physiological Anthropology, vol. 34, no. 1, p. 2, 2015.

[16] G. D. Johnson, B. Pavilonis, J. Caravanos, and J. Grassman, "Geo-spatial characterization of soil mercury and arsenic at a high-altitude Bolivian gold mine," Bulletin of Environmental Contamination and Toxicology, vol. 100, no. 2, pp. 259-264, 2018.
[17] M. Ning, H. Nailian, and L. Guoqing, "Evaluation of humanmachine effectiveness of plateau mine based on fuzzy analytic hierarchy process," Gold Science and Technology, vol. 6, pp. 871-878, 2019.

[18] D. Guo, G. Li, N. Hu, and J. Hou, "System dynamics analysis of man-machine efficacy in plateau mines," IEEE Access, vol. 9, pp. 18072-18084, 2021.

[19] Z. Wentao, H. Yuexin, and L. Yanjun, "Grinding technical efficiency prediction model based on matlab and particle swarm optimization," Journal of Northeastern University (Natural Science), vol. 40, pp. 95-98, 2019.

[20] U. Natarajan, R. Saravanan, and V. Periasamy, "Application of particle swarm optimisation in artificial neural network for the prediction of tool life," The International Journal of Advanced Manufacturing Technology, vol. 90, p. 2411, 2007.

[21] X. Niu, H. Wang, X. Li, and S. Hu, "Optimization of diesel engine responses prediction model based on neural network," Transactions of CSICE, vol. 36, pp. 561-568, 2018.

[22] S. Das, S. Mishra, and M. R. Senapati, "A novel model for stock price prediction using hybrid neural network," Journal of the Institution of Engineers (India): Series B, vol. 100, no. 4, p. 387, 2019.

[23] A. H. A. Othman, S. Kassim, R. B. Rosman, and N. H. B. Redzuan, "Prediction accuracy improvement for bitcoin market prices based on symmetric volatility information using artificial neural network approach," Journal of Revenue and Pricing Management, vol. 19, no. 5, pp. 314-330, 2020.

[24] F. B. Banadkooki, M. Ehteram, A. N. Ahmed et al., "Correction to: Suspended sediment load prediction using artificial neural network and ant lion optimization algorithm," Environmental Science and Pollution Research, vol. 27, no. 30, pp. 38117-38119, 2020.

[25] T. Y. Wu and K. W. Lei, "Correction to: prediction of surface roughness in milling process using vibration signal analysis and artificial neural network," The International Journal of Advanced Manufacturing Technology, vol. 102, no. 1-4, p. 315, 2019.

[26] L. Wang, B. Wu, Q. Zhu, and Y.-R. Zeng, "Forecasting monthly tourism demand using enhanced backpropagation neural network," Neural Processing Letters, vol. 52, no. 3, pp. 2607-2636, 2020.

[27] L. Dihong, G. Qun, and X. Xian, "Prediction of comprehensive performance of concrete based on BP neural network," Materials Reports, vol. 33, pp. 317-320, 2019.

[28] Z. Wenju, L. Yunxiang, and T. Deqiang, "Prediction of magnetic body top based on BP neural network," Oil Geophysical Prospecting, vol. 55, pp. 1139-1148, 2020.

[29] D. Liu, L. Wu, and Y. Yang, "A hybrid weight assignment model for urban underground space resources evaluation integrated with the weight of time dimension," Applied Sciences, vol. 10, no. 15, p. 5152, 2020.

[30] R. Yao, Y. Gao, and J. Xu, "Radio channel fingerprint model based on entropy weight method," in Proceedings of the 2016 Wireless and Optical Communication Conference (WOCC), Chengdu, China, May 2016.

[31] Q. Zhaoming, R. Gaofeng, and C. Fu-jiao, "Rock mass quality classification based on PCA and Fisher discrimination analysis," Rock and Soil Mechanics, vol. 37, pp. 427-432, 2016.

[32] L. Fraccaroli and F. Concli, "Introduction of open-source engineering tools for the structural modeling of a multilayer mountaineering ski under operation," Applied Sciences, vol. 10, no. 15, p. 5310, 2020. 
[33] R. Zhang, F. Nie, and X. Li, "Auto-weighted two-dimensional principal component analysis with robust outliers," in Proceedings of the 2017 IEEE International Conference on Acoustics, New Orleans, LA, USA, March 2017.

[34] J. Wu, Y. Hua, S. Yang, H. Qin, and H. Qin, "Speech enhancement using generative adversarial network by distilling knowledge from statistical method," Applied Sciences, vol. 9, no. 16, p. 3396, 2019.

[35] Y. Liu, Y. Wang, L. Deng et al., "A novel in situ compression method for CFD data based on generative adversarial network," Journal of Visualization, vol. 22, no. 1, pp. 95-108, 2019.

[36] J. Congcong, Intelligent Manufacturing System Performance Evaluation Modeling Based on Machine Learning, Zhejiang University of Technology, Hangzhou, China, 2019.

[37] F. Wang, G. Tian, X. Wang et al., "Application of genetic algorithm-back propagation for prediction of mercury speciation in combustion flue gas," Clean Technologies and Environmental Policy, vol. 18, no. 4, pp. 1211-1218, 2016.

[38] L. Qi, W. Feng, and W. Mingnian, "Study on mechanical efficiency of construction equipment in high altitude environment," Journal of Railway Science and Engineering, vol. 9, pp. 1974-1982, 2017.

[39] E. Brinksmeier, D. Meyer, A. G. Huesmann-Cordes, and C. Herrmann, "Metalworking fluids-mechanisms and performance," CIRP Annals, vol. 64, no. 2, pp. 605-628, 2015.

[40] F. Peng-fei and Z. Guan, "Prediction on geometrical characteristics of cermet laser cladding based on linear regression and neural network," Surface Technology, vol. 48, pp. 353-359, 2019. 